\title{
BMJ Open Understanding Dutch practice nurses' adherence to evidence-based smoking cessation guidelines and their needs for web-based adherence support: results from semistructured interviews
}

\author{
D de Ruijter, ${ }^{1}$ E S Smit, ${ }^{2}$ H de Vries, ${ }^{1}$ L Goossens, ${ }^{1}$ C Hoving ${ }^{1}$
}

To cite: de Ruijter $D$, Smit ES, de Vries $\mathrm{H}$, et al. Understanding Dutch practice nurses' adherence to evidence-based smoking cessation guidelines and their needs for web-based adherence support: results from semistructured interviews. BMJ Open 2017;7:e014154. doi:10.1136/bmjopen-2016014154

- Prepublication history and additional material is available. To view please visit the journal (http://dx.doi.org/ 10.1136/bmjopen-2016014154).

Received 6 September 2016 Revised 29 December 2016 Accepted 16 January 2017

CrossMark

${ }^{1}$ Department of Health Promotion, Care and Public Health Research Institute (CAPHRI), Maastricht University, Maastricht, The Netherlands

${ }^{2}$ Department of Communication Science, Amsterdam School of Communication Research (ASCoR), University of Amsterdam, Amsterdam, The Netherlands

\section{Correspondence to}

Dr C Hoving;

c.hoving@

maastrichtuniversity.nl

\section{ABSTRACT}

Objectives: Practice nurses in general practices suboptimally adhere to smoking cessation guidelines. Since the effectiveness of their smoking cessation support is greatest when full adherence to these guidelines is achieved, interventions need to be developed to improve practice nurses' guideline adherence, for example, by tailoring their content to adherence determinants. However, the sociocognitive determinants explaining adherence have not yet been investigated. Therefore, this qualitative needs assessment aimed to explore practice nurses' current counselling practices, as well as their sociocognitive beliefs related to their smoking cessation guideline adherence and their needs regarding web-based adherence support.

Setting: Primary care; general practices in the Netherlands.

Participants: 19 practice nurses, actively involved in smoking cessation counselling.

Methods: Semistructured individual interviews, based on the I-Change Model and the Diffusion of Innovations Theory, were conducted from May to September 2014. Data were systematically analysed using the Framework Method and considered reliable (к 0.77 ; \% agreement 99\%).

Results: Respondents felt able to be empathic and collaborative during smoking cessation consultations. They also reported psychological (eg, low self-efficacy to increase patient motivation and arranging adequate follow-up consultations) and practical barriers (eg, outdated information on quit support compensation and a perceived lack of high-quality trainings for practice nurses) to smoking cessation guideline adherence. Most respondents were interested in webbased adherence support to overcome these barriers.

Conclusions: Sociocognitive determinants influence practice nurses' smoking cessation guideline adherence. To improve their adherence, web-based tailored adherence support can provide practice nurses with personally relevant feedback tailored to individually perceived barriers to smoking cessation guideline adherence. More specifically, low self-efficacy levels can be increased by peer modelling (eg,

\section{Strengths and limitations of this study}

- Sociocognitive determinants of practice nurses' smoking cessation guideline adherence were not investigated yet.

- Interviews explored determinants of smoking cessation guideline adherence, both from a user's and from an innovation's perspective.

- Qualitative data were systematically coded and analysed using the Framework Method.

- Despite purposeful sampling, participants might differ from the 'average' practice nurse as they were possibly more involved in smoking cessation.

presenting narratives of colleagues) and up-to-date information can be presented online, enabling practice nurses to use it during patient consultations, resulting in more effective communication with their smoking patients.

Trial registration number: NTR4436; Pre-results.

\section{INTRODUCTION}

Globally, smoking continues to be the leading cause of preventable disease and premature death. ${ }^{12}$ Since $23 \%$ of the Dutch adult population still smokes, ${ }^{3}$ successful smoking cessation strategies are needed. Advice and counselling by a general practitioner (GP) is a cost-effective strategy to increase patients' quit rates. ${ }^{4}$ However, a shift in cessation counselling can be observed, where it is increasingly provided by trained practice nurses (PNs) in Dutch general practices. ${ }^{5} 6$ PNs are predominantly responsible for chronic patient care and lifestyle counselling, applying evidence-based guidelines. PNs are highly educated (ie, college degree) and employed in $80 \%$ of Dutch general practices. ${ }^{7}$ 
Regarding smoking cessation, PNs are trained to use one of several national smoking cessation guidelines that include similar counselling steps (figure 1) and of which the STIMEDIC guideline is the most recent. ${ }^{8}$ In the Netherlands, the 'Stop Smoking Partnership', an alliance of several parties involved in smoking cessation in the healthcare sector, is responsible for developing and regularly updating national smoking cessation guidelines. These guidelines can be used by healthcare professionals to structure consultations with smoking patients and full adherence to these evidence-based guidelines leads to more effective health communication and hence positively contributes to quality of smoking cessation care. ${ }^{9}{ }^{10}$ Adequate guideline adherence is also known to have stronger effects on patients' quit rates than a brief quit advice. ${ }^{11}$

However, PNs regularly report suboptimal adherence to smoking cessation guidelines, ${ }^{12-14}$ due to practical factors such as time constraints or the perception that most smoking patients are unwilling to quit. ${ }^{12} 1516$ Moreover, research has especially shown that sociocognitive determinants consistently influence guideline adherence among healthcare professionals. A study among cardiac nurses working in a hospital ${ }^{17}$ found significant positive correlations between self-efficacy expectations,

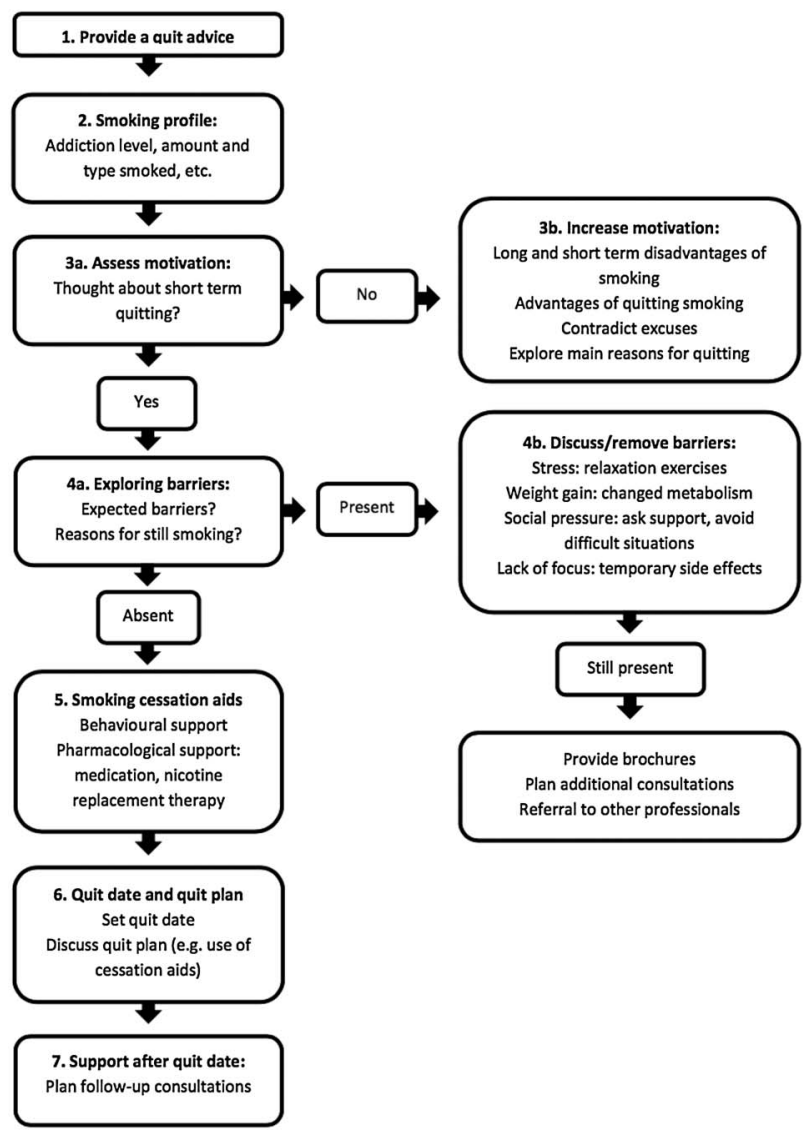

Figure 1 Flow chart Dutch smoking cessation guidelines (adapted from guideline treatment tobacco addiction revision $2009^{8}$ ). the perceived simplicity and advantages of the guideline, and intention towards continued use of a smoking cessation guideline. Relatedly, Segaar $e t a l^{13}$ concluded that a positive attitude, positive social influences and higher self-efficacy best explained adoption of the same guideline by cardiac nurses. Though these studies illustrate the importance of sociocognitive determinants in explaining smoking cessation guideline adoption and adherence, such data are not available for PNs working in general practices. It might be that different determinants influence the behaviour of PNs as they have different types of training and expertise, and work in a different setting with other types of patients compared with cardiac nurses. Therefore, it is important to investigate PNs' current counselling practices and the role of sociocognitive determinants in explaining their smoking cessation guideline adherence in a general practice setting.

Web-based computer tailored (CT) programmes have been shown to be able to effectively change various health behaviours and sociocognitive determinants among the general population and patient groups. ${ }^{18-21}$ CT programmes provide content adapted to characteristics (eg, demographic factors and sociocognitive determinants) of the individual user. ${ }^{22}$ A CT programme can, for instance, provide an individual with personal feedback about their behaviour, which is based on that individual's answers to a questionnaire. Consequently, individually tailored content is personally relevant and more likely to be read and remembered compared with more static tools such as leaflets or generic websites. ${ }^{22}$ Moreover, through web-based support, the intervention elements can be provided when and where $\mathrm{PNs}$ require them at a relatively low cost. ${ }^{23} 24$ Offering a minimally time-consuming approach enables $\mathrm{PNs}$ to integrate adherence support in their busy schedules, which was found to be important when asking nurses regarding their preferences for smoking cessation training. ${ }^{25} 26$ However, CT support has only been sparsely applied to increase smoking cessation guideline adherence among healthcare professionals and none have been offered in a web-based format. One study assessed the use of printed CT advice to increase smoking cessation counselling, ${ }^{27}$ demonstrating improved guideline implementation by physicians and increased patients' quit rates 6 months postintervention. Despite these promising results of printed CT advice among physicians, until now neither printed nor web-based CT support exists to stimulate smoking cessation guideline adherence among PNs.

To develop a web-based CT adherence support program for PNs, it is essential to assess PNs' current counselling practices and how determinants influence their adherence to smoking cessation guidelines, and to what extent. Furthermore, PNs' needs regarding the content and design of web-based support need to be assessed to ensure compatibility with their daily practice, as this contributes to higher exposure to an 
intervention's content. ${ }^{28}$ Therefore, we describe a qualitative needs assessment aimed at exploring PNs' current counselling practices, the sociocognitive determinants of their smoking cessation guideline adherence and their needs regarding web-based adherence support.

\section{METHODS}

\section{Study design}

Data were gathered by means of semistructured interviews with individual $\mathrm{PNs}(\mathrm{N}=19)$, because individual interviews yield more in-depth information than group interviews. $^{29}$ PNs provided oral informed consent. Interview recordings and verbatim transcriptions were anonymised and stored in a password-protected database. Evaluation of this study by the Medical Ethics Committee Atrium-Orbis-Zuyd (14-N-17) revealed that no medical ethical clearance for this study was needed according to the rules of the Medical Research Involving Human Subjects Act (WMO). The study is registered with the Dutch Trial Register (NTR4436).

\section{Participants and procedure}

Dutch PNs actively engaged in smoking cessation counselling in a general practice were recruited via telephone between May and September 2014. We invited a heterogenic sample of PNs to obtain diverse responses to the interview questions. Through purposeful sampling, we included PNs from (1) different geographical regions in the Netherlands; (2) different types of practices (urban and rural); and (3) practices with different sizes (in terms of patients and employees). Furthermore, recruited PNs differed in their (4) use of smoking cessation guidelines; (5) amount of working experience and (6) educational level. Recruitment continued until data saturation was reached; PNs did not introduce any new issues during the interviews at this point. Successfully contacted PNs who declined to participate $(n=7)$ mentioned a lack of time and interest in the study as main reasons. All participating PNs were rewarded with a $€ 10$ gift card.

Interviews were conducted at the PNs' workplace $(n=17)$ or via telephone $(n=2)$ and only the $\mathrm{PN}$ and one or two researchers (ie, DdR and LG) were present. No prior relationship between PNs and researchers was established before conducting the interviews. Both researchers had prior experience with conducting individual (ie, DdR) and group interviews (ie, LG). Before starting the interviews, PNs were informed about the aims of the interview. Interviews were audio-recorded and notes were taken to keep track of the issues and themes that were covered during each interview. Interviews lasted for 25-62 $\mathrm{min}(\mathrm{m}=43 \mathrm{~min})$.

\section{The interview guide}

A semistructured interview guide was developed based on the I-Change Model ${ }^{30}$ and the Diffusion of Innovations Theory, ${ }^{31}$ a combination which has been shown to be successful in explaining the implementation of smoking cessation interventions by primary healthcare professionals. ${ }^{132}$ In the interview guide, follow-up questions relating to PNs' current counselling practices and prompt questions regarding the STIMEDIC guideline were especially informed by the concepts attitude, social influence, self-efficacy, perceived barriers and skills from the I-Change Model. ${ }^{30}$ Questions relating to PNs' needs for web-based support (ie, the innovation) were guided by Rogers ${ }^{31}$ five innovation characteristics: the relative advantage, compatibility, complexity, trialability and observability of the innovation. Two primary care professionals engaged in smoking cessation counselling pretested the interview guide, which was adapted according to their feedback. The final interview guide (appendix 1) consisted of open-ended questions and prompts to encourage PNs to discuss their current smoking cessation practices, guideline adherence and desired programme characteristics.

Interviews were started with an assessment of PNs' personal (age, gender, personal smoking history and professional education) and work-related characteristics (years of experience as $\mathrm{PN}$, the number of practices employed in, and the size, location and type of the(se) general practice(s)). Subsequently, PNs were asked to describe their smoking cessation counselling routine and discuss barriers encountered during counselling. Third, the STIMEDIC smoking cessation guideline was introduced and PNs were asked to identify guideline steps that they would find difficult to perform and to describe situations they might find difficult to handle when applying these steps. Finally, PNs' needs were explored, including interest in and preferences regarding the content and design of web-based adherence support aiming to improve their smoking cessation counselling.

\section{Data analysis}

Data analysis using the Framework Method $^{33}$ was conducted using Nvivo (V.9). First, interviews were transcribed verbatim and two coders (ie, DdR and LG) familiarised themselves with the interview content by listening to audio records of the interviews and reading transcripts. Next, both coders independently developed a coding tree by analysing the same single transcript and the coding trees were compared for consistency; ${ }^{34}$ any inconsistencies were discussed and resolved. Then three similar rounds of independent coding of three additional transcripts resulted in a final coding tree consisting of multiple major and minor themes for every main interview question. This coding tree was perceived to cover all relevant information and agreed on by both coders. Subsequently, both coders independently applied the coding tree to the same five randomly selected interview transcripts and the intercoder reliability was assessed, resulting in a value for per cent agreement and Cohen's $\kappa$. Per cent agreement reflects the degree of similarity between coders in assigning the same code to the same piece of text ${ }^{34}$ and a coefficient 
of $\geq 90 \%$ is considered acceptable. ${ }^{35} 36$ Cohen's $\kappa$ takes into account that agreement between coders might occur due to chance and is therefore a more conservative coefficient; ${ }^{35}$ a $\kappa$ of $\geq 0.70$ is considered acceptable. ${ }^{35}$ It was determined that the intercoder agreement was sufficiently high ( $\%$ agreement $99 \%$; Cohen's $\kappa$ 0.72 ). Nonetheless, coding inconsistencies were again discussed and resolved. Thereafter, the first coder (ie, DdR) completed coding the remaining transcripts $(n=14)$, whereas the second coder (ie, LG) only coded the final $10 \%$ of the transcripts $(n=2)$. This revealed an improved intercoder reliability of 99\% (\% agreement) and 0.77 (Cohen's $\kappa)$. Once all transcripts were coded, themes were grouped together and data were clustered based on their importance. Finally, data clusters were interpreted by looking for patterns and identifying answers to the research questions.

\section{RESULTS}

Participating PNs were on average 46.1 years old and mostly female; the majority had never smoked (table 1 ). All PNs had obtained a Bachelor's degree in nursing, while some were additionally trained in pulmonary or diabetic patient care. PNs' mean work experience was 8.3 years; they were mostly employed at urban practices and reported a mean number of 8.4 employees (range 4-20) and a patient population of nearly 4500 patients (range 500-12 000) per practice.

\section{Current smoking cessation counselling practices}

All PNs reported using a national guideline to structure their counselling, but described varying levels of guideline adherence. Smoking cessation counselling would usually be initiated by GPs' patient referral to a PN (ie, GP provides a short quit advice, then refers to $\mathrm{PN}$ ) or by PNs themselves giving patients a brief quit advice, especially 'people who visit with lung problems or diabetes'.

After a quit advice, additional counselling was predominantly conducted by PNs, mostly by first assessing their patients' smoking profile (eg, amount smoked, smoking pattern, addiction level) during an intake interview.

Most PNs also reported assessing patients' motivation to quit, but more than half indicated discontinuing counselling if "they [patients] are not internally motivated...Becoming internally motivated often takes longer than quitting itself." Those PNs attempting to actively increase their patients' motivation used techniques like reflecting on patients' intrinsic reasons for quitting and providing patients with homework assignments.

When they go home I ask [the patient] to write down their personal pros and cons of smoking... When they come back we especially discuss alternatives for the pros of smoking.
Table 1 Descriptive characteristics of participating PNs $(\mathrm{N}=19)$

\section{Personal characteristics}

\begin{tabular}{lc}
\hline Mean age in years (SD) & $46.1(9.2)$ \\
Female (\%) & $17(89.5 \%)$ \\
Smoking status & $11(57.9 \%)$ \\
$\quad$ Never smoked (\%) & $8(42.1 \%)$ \\
$\quad$ Ex-smoker (\%) & \\
Professional education & $19(100 \%)$ \\
Postbachelor PN training (\%) & $3(15.8 \%)$ \\
Specialised diabetes nurse (\%) & $4(21.1 \%)$ \\
Specialised pulmonary nurse (\%) & $8.3(4.0)$
\end{tabular}

years (SD)

\begin{tabular}{lr}
\hline Practice characteristics & \\
\hline Urban practice location (\%) & $12(63.2 \%)$ \\
Mean number of employees in & $8.4(4.4)$ \\
practice (SD) & \\
Mean patient visits per year* (SD) & $4478.4(3016.4)$ \\
\hline & Adherence by \\
Counselling elements & PNs (n)† \\
\hline Provide a quit advice & $10(52.6 \%)$ \\
Assess patients' smoking profile & $18(94.7 \%)$ \\
Assess patients' motivation & $18(94.7 \%)$ \\
Increase patients' motivation & $7(36.8 \%)$ \\
Assess patients' barriers & $15(78.9 \%)$ \\
Remove patients' barriers & $7(36.8 \%)$ \\
Discuss use of cessation aids & $16(84.2 \%)$ \\
Develop a quit plan & $16(84.2 \%)$ \\
Follow-up after the quit date & $18(94.7 \%)$ \\
\hline *Standardised practice capacity: 2168 patients per full-time \\
general practitioner. \\
†The data represent the number (and percentage) of adherent PNs. \\
PN, practice nurse.
\end{tabular}

The majority of PNs reported assessing patients' barriers for quitting, but less than half also attempted to remove present barriers, which was usually done by discussing distraction strategies to deal with craving.

Craving for cigarettes only lasts a minute...I especially discuss distraction strategies to deal with this minute.

Most PNs discussed the use of cessation aids (eg, nicotine replacement therapy, medication, group counselling), especially focusing on their working mechanisms and possible side effects. Patients' preferences often influenced the decision to prescribe a particular cessation aid.

Most patients know about Champix (varenicline) and specifically request it because they have heard about it from others.

Subsequently, the majority of PNs reported developing a quit plan with their patients, including a quit date, the use of cessation aids and follow-up appointments. They described that the duration, frequency and format of follow-up appointments after the quit date strongly 
depend on patients' preferences and their level of motivation, ranging from multiple face-to-face appointments to a single telephone appointment.

First, patients often did not return...By planning follow-up appointments more frequently...I have more success in [helping them] quitting smoking.

Concerning their role as a smoking cessation counsellor, PNs perceived themselves able to 'adapt counselling to a (patient's) personal situation', to stimulate patients to make their own decisions by weighing personal pros and cons, and to provide patients with relevant information 'which is evidence-based' about the disadvantages of smoking.

\section{Experienced barriers during counselling}

PNs found it difficult to handle unmotivated patients, because they struggle to decide how much they should try to increase their patients' motivation. Since they believed that the success of their counselling mainly depends on their patients' level of knowledge of and awareness about possibilities for smoking cessation and not so much on their own efforts to enhance patients' motivation, PNs sometimes decided to only provide patients with awareness-enhancing information.

If a patient reports not to need counseling, I experience that it [counseling] has no effect. I am glad when they [patients] agree to take home some information.

Several PNs described that this often happens after GPs directly referred patients without first assessing patients' motivation or providing a brief quit advice. They believed GPs' advice could be motivation-enhancing for patients, but felt 'it (GPs' quit advice) does not happen enough'.

If the counselling process was continued, PNs did not attempt to remove patients' barriers when their patients' motivation was low, as they perceived that 'unmotivated patients should first work on their motivation themselves'. PNs generally believed that a sufficient level of motivation is first needed (before dealing with the presence of barriers), but that achieving this is mainly the patient's own responsibility.

Regarding follow-up after the quit date, PNs felt that smoking cessation guidelines lack detailed instructions on which topics to discuss during follow-up appointments.

You call them [patients] or they visit the practice and of course you talk about their quit attempt, but there is no specific plan.

Moreover, they described that patients do not always perceive the need for follow-up and regularly skip or cancel scheduled appointments, making it difficult for PNs to 'get them (patients) to return to practice' for follow-up and to determine their success rate, since 'it is their (the patients') own responsibility'.
Several PNs reported difficulties staying up to date regarding smoking cessation counselling as they perceived a low supply of high-quality training classes. Furthermore, associated monetary and time investments were considered barriers for attending classes that do exist. Moreover, most PNs reported troubles with staying informed about compensation of counselling by different health insurances, as they all have their own regulations (eg, a maximum number of consultations), which change regularly. Several PNs reported that 'online medication orders are not delivered on time', which means that 'a well-prepared quit attempt falls to pieces'. Obtaining reliable information about both health insurances and ordering online prescriptions was described as 'very time-consuming and sometimes leading to confusion'.

Barriers for providing patients with educational materials included a lack of time and high-quality brochures, containing educational and visually attractive content. Moreover, patients were perceived as often not using provided materials and consequently lacking awareness of and knowledge about smoking cessation aids and counselling.

It is not the case that you take a single pill of Champix and suddenly do not like cigarettes anymore...Sometimes it is necessary to adjust this expectation.

\section{Needs regarding adherence support}

Most PNs stated interest in web-based support to improve their smoking cessation counselling. Several PNs were already familiar with other web-based programmes for primary care professionals and positively evaluated these programmes. They expressed interest in being able to "browse through a program and choose only those things that I can personally apply during counseling."

Regarding compatibility, PNs expected the content to be up to date, individually relevant and applicable in practice. Moreover, a programme should be easy to use (low complexity) and require little time investment.

I want to know the latest developments about smoking cessation... and easily retrieve them from a web-based program, without having to search through databases.

Long questionnaires and extensive texts were perceived as potential disadvantages and availability after working hours was perceived as a potential advantage of web-based support. Furthermore, PNs wanted a programme free to visit and revisit at their convenience without obligations (high trialability). Visually attractive content (ie, images or videos) and experiences of other PNs were preferred over a text-only form of support (relative advantage).

Experiences of others would be interesting...[]...so you know what was successful and which strategies did not work. 
Most PNs also expressed a need for printable patientcentred educational materials to use during counselling, such as an overview of their patients' counselling trajectory or a timeline depicting physical advantages of quitting smoking.

The information should be suitable for patients...simple and clear, without complicated terminology. Preferably a combination of text and figures.

\section{DISCUSSION AND CONCLUSION}

Our results add a novel perspective to the scientific literature on health communication by describing PNs' smoking cessation counselling practices, determinants of their adherence to evidence-based guidelines as well as their needs regarding a web-based adherence support programme. Results indicate that while all PNs reported the use of an evidence-based guideline, their guideline adherence was suboptimal, which was influenced by psychological and practical barriers. Most PNs showed interest in web-based adherence support that could help them to overcome these barriers and reported specific needs that could be addressed to ensure a programme's optimal compatibility to their work situation.

\section{Discussion}

Despite specific difficulties that PNs perceive regarding full adherence to smoking cessation guidelines, they also reported being confident that they could adapt their counselling to the situation of smoking patients, to stay close to a patient's preferences and beliefs with respect to a quit attempt. In other words, PNs felt able to provide empathic counselling in a collaborative way, which are core elements of motivational interviewing, ${ }^{37}$ and hence an important competence to possess and apply during smoking cessation consultations.

Nevertheless, PNs also reported psychological and practical barriers regarding their adherence to evidencebased guidelines. A psychological barrier to guideline adherence was PNs' perceived inability to motivate patients to quit smoking; they reported to rather provide awareness-enhancing information than to actively try to increase patients' motivation, a finding also described in studies investigating nurses' adherence to motivational interviewing techniques. ${ }^{38} 39$ PNs' perceived inability to motivate smokers could indicate a lack of self-efficacy, which was also found in other studies investigating determinants of nurses' use of guidelines or protocols. ${ }^{17} 40$ Low self-efficacy could possibly originate from PNs' perceptions that they are of minor influence on patients' intrinsic motivation (ie, low confidence that counselling will make a difference). Relatedly, PNs indicated that they often did not actively try to remove barriers when dealing with unmotivated patients. However, patients' lack of intrinsic motivation could be the result of seeing many barriers for quitting and hence not discussing them could be a missed opportunity to increase patients' motivation to quit. ${ }^{41}$ Furthermore, PNs reported difficulties in ensuring their patients' presence at follow-up appointments. This potentially originates from their perception that it is their patients' responsibility to show up. Consequently, they do not repeatedly contact their patients about missed appointments, as was also found in a previous study among primary care professionals' smoking cessation counselling practices. ${ }^{16}$

Concerning practical barriers for guideline adherence, PNs perceived a lack of high-quality smoking cessation training classes and reported a lack of uniformity about health insurances' compensation policies. Accordingly, PNs had difficulties providing patients with personally relevant and up-to-date information. Additionally, retrieving information about such topics was considered to be very time-consuming, though necessary to enable patients to make an informed choice about their quit attempt. Such time-consuming tasks may cause time constraints regarding actual cessation counselling activities, a commonly mentioned barrier among primary care professionals, ${ }^{15}{ }^{42}$ and consequently interfere with PNs' adherence to smoking cessation guidelines.

When considering solutions for the identified barriers, most PNs were interested in web-based adherence support. To overcome these barriers, PNs expressed the need for tailored support (ie, instead of offering generic information), compatible with their situation and with low complexity and high trialability. PNs' perceived accessibility after office hours as a potential advantage of the web-based nature of such a programme. These findings are in line with previous studies that related innovation characteristics to increased adoption and implementation of novel interventions by nurses. ${ }^{43} 44$ Furthermore, PNs would like to be supported with practical tools (eg, printable patient materials) to apply during counselling and hence improve effective communication about smoking cessation.

\section{Practice implications}

The development of web-based adherence support seems suitable to help PNs to overcome psychological and practical barriers to improve their smoking cessation guideline adherence. That is, a web-based CT programme could provide PNs with personal feedback about performing specific counselling elements they find difficult to apply, based on evidence-based methodologies to change sociocognitive determinants as described elsewhere. ${ }^{45}$ For instance, role modelling could be used to increase self-efficacy, which can be operationalised by presenting PNs with narratives of peers (ie, fellow PNs) via a web-based programme. ${ }^{46}$ Targeting sociocognitive determinants through a tailored web-based support programme is a well-established method to successfully influence these determinants and ultimately behaviour change. ${ }^{22} 47$

Furthermore, tailored feedback can be supplemented with up-to-date information about smoking cessation counselling in primary care, like an overview of available 
training classes or health insurances' compensation policies. This could reduce PNs' time needed to find this information and consequently increase their time spent on counselling. Finally, PNs can be supported with practical tools, like visually attractive educational materials for lower educated patients and patients with different cultural backgrounds. Such strategies potentially enable PNs to optimally adhere to evidence-based guidelines and subsequently promote the quality of their smoking cessation care.

\section{Strengths and limitations}

A strength of our study was that the interview guide was based on the I-Change Model $^{30}$ and the Diffusion of Innovations Theory. ${ }^{31}$ This enabled us to explore determinants of smoking cessation guideline adherence from both the users' (ie, PNs) and the innovation's (ie, webbased adherence support) perspective, a combination which has previously been associated with nurses' adoption and implementation behaviour of intervention programmes. ${ }^{13} 43$ Second, owing to the semistructured nature of the interview guide, PNs had ample opportunity to elaborate on their experiences during smoking cessation counselling, providing a vast amount of data per interview. Moreover, the interviews enabled us to assess PNs' individual preferences for web-based adherence support to optimise such a programme's compatibility. ${ }^{48}$ Third, qualitative data were systematically analysed using the Framework Method, ensuring optimal coverage of relevant content through the development of an extensive coding tree. ${ }^{33}$ Moreover, intercoder reliability was high, indicating that the coding was sufficiently objective and valid.

A limitation of the study was that PNs interested in smoking cessation research may have been more likely to participate in the interviews than non-interested PNs. This may have threatened the generalisability of the results to the total population of PNs. Nonetheless, by purposeful sampling, we recruited a heterogenic group of PNs who provided a great variability of responses. Moreover, data saturation occurred within the 19 interviews conducted.

\section{Conclusion}

Dutch PNs experienced several barriers to completing smoking cessation guideline adherence. They reported low self-efficacy to motivate patients and to remove patients' barriers to quit, and struggled with successfully organising patient follow-up. Moreover, a perceived lack of smoking cessation training classes and uniform insurance policies was identified as practical barriers for optimal guideline adherence. PNs were interested in web-based adherence support to overcome these barriers, especially if a web-based programme is easy to use, free to (re)visit and compatible with their current smoking cessation practices. Taking PNs' needs into account when developing a programme is likely to stimulate the programme's usage and maximise exposure to its content, making it more likely to be effective in improving PNs' smoking cessation guideline adherence.

Acknowledgements The authors wish to thank all participating practice nurses for their time. They would also like to thank the research assistants for assisting with transcribing the interview audiotapes.

Contributors All authors significantly contributed to the drafting of this manuscript. ESS, HdV andCH developed the proposal for the present research. DdR was responsible for conducting and reporting on the research. $\mathrm{LG}$ assisted in data collection and analysis and ESS, HdV and $\mathrm{CH}$ supervised the process and provided feedback.

Funding This work was supported by the Dutch Cancer Society, grant number UM2013-6107.

Competing interests None declared.

Ethics approval Medical Ethics Committee Atrium-Orbis-Zuyd.

Provenance and peer review Not commissioned; externally peer reviewed.

Data sharing statement Requests to share anonymised data will be considered. Please contact the first author at d.deruijter@maastrichtuniversity. $\mathrm{nl}$

Open Access This is an Open Access article distributed in accordance with the Creative Commons Attribution Non Commercial (CC BY-NC 4.0) license, which permits others to distribute, remix, adapt, build upon this work noncommercially, and license their derivative works on different terms, provided the original work is properly cited and the use is non-commercial. See: http:// creativecommons.org/licenses/by-nc/4.0/

\section{REFERENCES}

1. WHO. WHO report on the global tobacco epidemic 2013: enforcing bans on tobacco advertising, promotion and sponsorship. Geneva: World Health Organization, 2013.

2. USDHHS. The health consequences of smoking-50 years of progress. A report of the surgeon general. Atlanta, GA: USA. Department of Health and Human Services, Centers for Disease Control and Prevention, National Center for Chronic Disease Prevention and Health Promotion, Office on Smoking and Health, 2014.

3. RIVM. Volksgezondheid Toekomst Verkenning (VTV) 'Een gezonder Nederland'. Bilthoven: 2014.

4. Stead LF, Buitrago D, Preciado N, et al. Physician advice for smoking cessation. Cochrane Database Syst Rev 2013;(5): CD000165.

5. Nederlands Huisartsen Genootschap \& Landelijke Huisarsten Vereniging. NHG/LHV Standpunt: Het (ondersteunend) Team in de huisartsenvoorziening. Utrecht: Nederlands Huisartsen Genootschap and Landelijke Huisartsen Vereniging, 2011.

6. Freund T, Everett C, Griffiths P, et al. Skill mix, roles and remuneration in the primary care workforce: who are the healthcare professionals in the primary care teams across the world? Int $J$ Nurs Stud 2015;52:727-43.

7. Heiligers PJM, Noordman J, Korevaar JC, et al. Kennisvraag: praktijkondersteuners in de huisartspraktijk (POH's), klaar voor de toekomst? Utrecht: NIVEL, 2012.

8. Stop Smoking Partnership. Guideline treatment tobacco addiction [Richtlijn behandeling van tabaksverslaving]. Alphen aan den Rijn, The Netherlands: 2009.

9. Miller M, Kearney N. Guidelines for clinical practice: development, dissemination and implementation. Int J Nurs Stud 2004;41:813-21.

10. Woolf SH, Grol R, Hutchinson A, et al. Clinical guidelines: potential benefits, limitations, and harms of clinical guidelines. BMJ 1999;318:527-30.

11. Rice VH, Hartmann-Boyce J, Stead LF. Nursing interventions for smoking cessation. Cochrane Database Syst Rev 2013;(8): CD001188.

12. Whitehead D, Zucker SB, Stone J. Tobacco cessation education for advanced practice nurses. Nurse Educ 2014;39:252-5.

13. Segaar D, Willemsen MC, Bolman C, et al. Nurse adherence to a minimal-contact smoking cessation intervention on cardiac wards. Res Nurs Health 2007;30:429-44.

14. Glasgow RE, Lichtenstein E, Marcus AC. Why don't we see more translation of health promotion research to practice? Rethinking the 
efficacy-to-effectiveness transition. Am J Public Health 2003;93:1261-7.

15. Studts JL, Flynn SM, Dill TC, et al. Nurse practitioners' knowledge, attitudes, and clinical practices regarding treatment of tobacco use and dependence. J Nurse Pract 2010;6:212-19.

16. van Rossem C, Spigt MG, Kleijsen JR, et al. Smoking cessation in primary care: exploration of barriers and solutions in current daily practice from the perspective of smokers and healthcare professionals. Eur J Gen Pract 2015;21:1-7.

17. Bolman $\mathrm{C}$, de Vries $\mathrm{H}$, Mesters I. Factors determining cardiac nurses' intentions to continue using a smoking cessation protocol. Heart Lung 2002;31:15-24.

18. Lustria MLA, Noar SM, Cortese J, et al. A meta-analysis of web-delivered tailored health behavior change interventions. $J$ Health Commun 2013;18:1039-69.

19. Krebs P, Prochaska JO, Rossi JS. A meta-analysis of computer-tailored interventions for health behavior change. Prev Med 2010;51:214-21.

20. Kohl LFM, Crutzen R, de Vries NK. Online prevention aimed a lifestyle behaviors: a systematic review of reviews. J Med Internet Res 2013;15:e146.

21. Civljak M, Stead LF, Hartmann-Boyce J, et al. Internet-based interventions for smoking cessation. Cochrane Database Syst Rev 2013;(7):CD007078.

22. Kreuter MW, Wray RJ. Tailored and targeted health communication: strategies for enhancing information relevance. Am J Health Behav 2003;27(Suppl 3):S227-32

23. Griffiths F, Lindenmeyer A, Powell J, et al. Why are health care interventions delivered over the internet? A systematic review of the published literature. J Med Internet Res 2006;8:e10.

24. Tate DF, Finkelstein EA, Khavjou O, et al. Cost Effectiveness of Internet Interventions: review and recommendations. Ann Behav Med 2009;38:40-5

25. Smith PM, Sellick SM, Spadoni MM. Tobacco cessation Clinical Practice Guideline use by rural and urban hospital nurses: a pre-implementation needs assessment. BMC Nurs 2012;11:6.

26. Katz DA, Paez MW, Reisinger HS, et al. Implementation of smoking cessation guidelines in the emergency department: a qualitative study of staff perceptions. Addict Sci Clin Pract 2014;9:1.

27. Unrod M, Smith M, Spring B, et al. Randomized controlled trial of a computer-based, tailored intervention to increase smoking cessation counseling by primary care physicians. J Gen Intern Med 2007;22:478-84

28. Schneider F, van Osch L, de Vries H. Identifying factors for optimal development of health-related websites: a Delphi study among experts and potential future users. J Med Internet Res 2012;14:e18.

29. Bourgeault I, Dingwall R, Vries RD. The SAGE handbook of qualitative methods in health research. London: SAGE Publications Ltd, 2010:186.

30. de Vries $\mathrm{H}$, Mudde A, Leijs I, et al. The European Smoking Prevention Framework Approach (EFSA): an example of integral prevention. Health Educ Res 2003;18:611-26.

31. Rogers EM. Diffusion of innovations. 5th edn. New York: Free Press, 2003.
32. Smit ES, de Vries $\mathrm{H}$, Hoving $\mathrm{C}$. The PAS study: a randomized controlled trial evaluating the effectiveness of a web-based multiple tailored smoking cessation programme and tailored counselling by practice nurses. Contemp Clin Trials 2010;31:251-8.

33. Gale NK, Heath G, Cameron E, et al. Using the framework method for the analysis of qualitative data in multi-disciplinary health research. BMC Med Res Methodol 2013;13:117.

34. Campbell JL, Quincy C, Osserman J, et al. Coding in-depth semistructured interviews: problems of unitization and intercoder reliability and agreement. Sociol Methods Res 2013;42:294-320.

35. Lombard M, Snyder-Duch J, Bracken CC. Content analysis in mass communication: assessment and reporting of intercoder reliability. Hum Commun Res 2002;28:587-604.

36. Krippendorff K. Reliability in content analysis. Hum Commun Res 2004;30:411-33.

37. Copeland L, McNamara R, Kelson M, et al. Mechanisms of change within motivational interviewing in relation to health behaviors outcomes: a systematic review. Patient Educ Couns 2015;98:401-11.

38. Efraimsson EO, Fossum B, Ehrenberg A, et al. Use of motivational interviewing in smoking cessation at nurse-led chronic obstructive pulmonary disease clinics. J Adv Nurs 2012;68:767-82.

39. Noordman J, van Lee I, Nielen $\mathrm{M}$, et al. Do trained practice nurses apply motivational interviewing techniques in primary care consultations? J Clin Med Res 2012;4:393-401.

40. Cassista J, Payne-Gagnon J, Martel B, et al. Applying theory to understand and modify nurse intention to adhere to recommendations regarding the use of filter needles: an intervention mapping approach. Nurs Res Pract 2014;2014:356153.

41. Bundy C. Changing behaviour: using motivational interviewing techniques. J R Soc Med 2004;97(Suppl 44):43-7.

42. Berndt NC, Bolman $\mathrm{C}$, de Vries $\mathrm{H}$, et al. Smoking cessation treatment practices: recommendations for improved adoption on cardiology wards. J Cardiovasc Nurs 2013;28:35-47.

43. Smit ES, de Vries H, Hoving C. Determinants of practice nurses' intention to implement a new smoking cessation intervention: the importance of attitude and innovation characteristics. J Adv Nurs 2013;69:2665-74

44. van der Weide M, Smits J. Adoption of innovations by specialised nurses: personal, work and organisational characteristics. Health Policy 2004;68:81-92.

45. de Ruijter $\mathrm{D}$, Smit ES, de Vries $\mathrm{H}$, et al Web-based computertailoring for practice nurses aimed to improve smoking cessation guideline adherence: a study protocol for a randomized controlled effectiveness trial. Contemp Clin Trials 2016;48:125-32.

46. Kok G, Schaalma H, Ruiter RA, et al. Intervention mapping: protocol for applying health psychology theory to prevention programmes. $J$ Health Psychol 2004:9:85-98.

47. Lustria ML, Cortese J, Noar SM, et al. Computer-tailored health interventions delivered over the Web: review and analysis of key components. Patient Educ Couns 2009;74:156-73.

48. Avis JL, van Mierlo T, Fournier R, et al. Lessons learned from using focus groups to refine digital interventions. JMIR Res Protoc 2015;4:e95. 